\title{
El ordenador como instrumento de la mente
}

\section{David R. Olson}
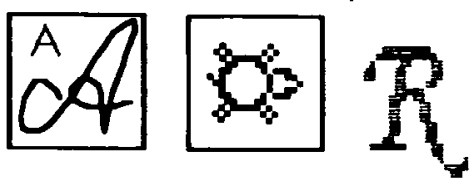

Si cada instrumento psicológico - por utilizar la expresión de Vygotski- o cada extensión de la mente - empleando la de McLuban-determina un modo de pensar y de ser inteligente, las generaciones que boy coinciden en la clase, maestros y alumnos, se enfrentan a una superposición y convergencia nunca vista en la bistoria de nuestros instrumentos para la mente. ¿Cuáles son las caracteristicas y consecuencias de este hecho? D. Olson responde a esta pregunta, centrándola especialmente en la lectoescritura y el ordenador.

\section{LOS ORDENADORES COMO HERRAMIENTAS DEL INTELECTO}

Voy a desarrollar en este artículo la idea de que la inteligencia no es simplemente una cualidad básica de la mente, sino un producto de la relación entre las estructuras de la mente y las «tecnologías del intelecto», como Goody denominaba a los mecanismos culturales que la persona utiliza para pensar (Goody, 1977; Olson, 1976). Estos mecanismos varían según las diferentes culturas, pero todos incluyen sistemas de símbolos tales como representaciones numéricas, representaciones espaciales, -incluidos mapas y diagramas-; y representaciones del lenguaje, incluidas la escritura y la imprenta, medios éstos de expresión y comunicación generalizados.

$\mathrm{Si}$ hemos de entender la inteligencia en función de estas tecnologías, es importante considerar en qué medida la creciente dependencia de la tecnología informática modificará el intelecto. Si la competencia en la lengua escrita es básica para comprender la inteligencia en una sociedad alfabetizada

"Computers as tools of intellect" Educational Researcher, 1985, mayo, 5-8. Reproducido con autorización del autor y de la American Educational Research Ass. Washington, D. C. (C) Traducción al castellano, CLEE, 1989 (Traducción de Begoña Jiménez). 
(la sociedad que ha predominado desde el siglo XVI), ¿ en qué consiste la inteligencia en un mundo informatizado (el mundo del siglo $\mathrm{XX}$ )?

No seamos tan optimistas como para pensar que la respuesta es fácil o rápida. Sólo en los últimos 25 años hemos empezado a comprender la importancia de la alfabetización en la cultura y en el conocimiento, a pesar de que al menos una parte de nuestra sociedad ha estado alfabetizada desde hace tres siglos y medio. Como McLuhan señala en 1962, el afán puesto en el empleo de la técnica no nos ha dejado tiempo para considerar sus implicaciones. Quizá merezca la pena sin embargo aplicar las estrategias diseñadas en la investigación de las implicaciones de la alfabetización al problema de las implicaciones cognitivas del trabajo con ordenadores.

El ordenador podría modificar las funciones mentales de dos distintas maneras: una, alterando la base de conocimientos de la persona; otra, alterando las operaciones aplicables a dicha base de conocimientos. Aunque, como iremos viendo, la solución al problema no es tan sencilla, estas dos categorías constituyen un primer paso a la hora de abordar las consecuencias del empleo de ordenadores.

\section{Modificación de la base de conocimientos}

Como ya han señalado algunos autores (Havelock, 1982; Olson y Torrance, 1983; Ong, 1982), la escritura hizo que el lenguaje pasara, de ser un medio efímero de comunicación, a ser un objeto visible y permanente, la palabra escrita. Una vez que la palabra escrita es vista como objeto, la palabra hablada puede asimismo ser tratada como un objeto; en suma, el lenguaje se convierte en objeto. La escritura mantiene las palabras - lo que es "dicho"-, pero no mantiene el «significado" si por tal entendemos las intenciones del hablante o del escritor. Así, la escritura crea un problema de significado; esto es, mantener el lenguaje como un objeto plantea lo que podemos denominar el problema de la interpretación (conciencia de la ambigüedad del lenguaje, el problema de intención del significado/interpretación del significado, el problema de cuando uno está afirmando, declarando, pidiendo o simplemente conjeturando, etc.). Cualquier intento de solucionar estos problemas exige prestar una rigurosa atención a las propias palabras y a sus "significados literales" - la elaboración de conceptos- y exige términos que diferencian dichos significados. Conceptos como pretender, interpretar, inferir, así como conceptos de estado mental y de acto de habla tan cruciales para los análisis actuales del lenguaje y el pensamiento.

El desarrollo de una tradición escrita supuso la invención de tales conceptos. Ser hoy letrado supone manejar estas distinciones conceptuales a la hora de enfrentarse a los problemas de interpretación de la lengua escrita. Armados de estas distinciones, una persona está en posición de dividir en dos partes cualquier elocución leída $\mathbf{u}$ oída: lo que se ha dicho y la interpretación que ella le atribuye. De esta forma, es menos probable que atribuyamos nuestras interpretaciones a las intenciones del hablante. Reconoceremos la ambigüedad residual de la mayor parte del lenguaje, explotándola en ciertos tipos de discurso y de escritura, como por ejemplo la poesía, y liminándola en otros, como la prosa científica.

Los juegos de comunicación estudiados por Robinson y Robinson (1982), y Robinson, Goelman y Olson (1983) ilustran claramente la difi- 
cultad que los niños tienen para diferenciar lo que se ha dicho de las interpretaciones que uno puede hacer. Los niños no parecen darse cuenta de que la interpretación que ellos dan a una frase puede ser distinta de la interpretación que le asigna otra persona, es decir, fracasan en el reconocimiento de la ambigüedad. Para ellos la "opción por defecto" es que los demás hagan idénticas interpretaciones e inferencias cuando oyen o ven algo.

Antes de aprender a leer, los niños no reconocen la ambigüedad del lenguaje - su carácter abierto a la interpretación-, y tampoco parecen reconocerla algunas sociedades no alfabetizadas. En un sobresaliente trabajo sobre brujería y oráculos entre los Azande, Evans-Pritchard (1937) escribía lo siguiente sobre estos problemas de interpretación del lenguaje: «Todo lo que diga una persona acusada de brujería es interpretado en sentido diferente al que ella intentaba dar a sus palabras" (pág. 133). A Evams-Pritchard le sorprendía que dichas «interpretaciones» se atribuyeran directamente al hablante y se utilizaran para probar su culpabilidad. Desde luego, no eran interpretaciones en el sentido que nosotros entendemos el término cuando nos referimos a la lengua escrita; no se consideraban producto del oyente, como lo consideraríamos nosotros, sino que se atribuía al hablante una interpretación inventada por el oyente. Los azande interpretan su lengua exactamente igual que cualquier hablante de otra lengua, pero no distinguen sus interpretaciones de lo que el hablante realmente dice o pretende decir.

Ese es precisamente el problema que tienen los niños en nuestra sociedad con la ambigüedad del lenguaje. Dan por supuesto que la intención o interpretación es exactamente lo que el hablante ha dicho. Cuando llegan a una interpretación, están convencidos de que la frase no podía ser interpretada de otro modo (Beal y Flavell, en preparación).

Estas distinciones conceptuales no sólo son útiles para interpretar textos, sino que también son vitales para comprender las intenciones del hablante o del agente en relación con sus enunciados o acciones, y son vitales para adoptar lo que podría denominarse una postura teórica frente a la naturaleza. Sin embargo, la propia distinción tiene sus raíces en la alfabetización, tal como recientemente he intentado mostrar (Olson, en preparación).

¿Qué ocurre con los ordenadores y el conocimiento? No me parece que el ordenador desplace estas distinciones conceptuales: antes bien, las impone con mayor severidad. En la lengua escrita, es de suma importancia reconocer que el significado de una frase puede no corresponderse con lo que el escritor ha querido comunicar con ella; el lector debe depurar lo que se dice a fin de obtener una representación explícita y precisa del significado o de la intención.

Si para la lengua es importante que el significado esté explícito, para el ordenador es crucial. Los ordenadore sólo pueden "decodificar» el significado ya explícito en el texto, no pueden «interpretar" ese texto. Todas las interpretaciones que se quieran otorgar deben ir explícitamente señalizadas en el programa. Los enunciados ambiguos no son computables.

¿Repercute este requisito de exactitud en la cognición? Entra dentro de lo razonable esperar que ayudará a identificar con mayor nitidez lo que es representado en el lenguaje y lo que es añadido por el lector o el oyente al crear una interpretación de dicha representación. Una comprensión clara de esta distinción permitiría a programadores, escritores y hablantes con- 
feccionar sus enunciados de manera que estén menos sujetos a interpretación, y podría someterse a comprobación empírica si esta posibilidad formentaría la precisión de las expresiones.

Los ordenadores imponen con severidad la distinción entre lo que un texto dice y la interpretación que uno le atribuye. Gran parte de la investigación informática se ha dedicado a diseñar programas capaces de remediar estos problemas de interpretación mediante la creación de contextos más ricos para las frases que aparecen. Las teorías de scripts (guiones) y frames (marcos) son un ejemplo de ello. Por supuesto, un ordenador no puede llegar a interpretaciones personales, subjetivas y privadas, sino sólo a interpretaciones erróneas. Un ordenador trabaja unicamente con representaciones formales, explícitas y con operaciones sobre dichas representaciones, incluso si el contenido a interpretar es muy sofisticado.

Los ordenadores proporcionan una vía prometedora e interesante para ayudar a los niños a enfrentarse con el problema de la ambigüedad del lenguaje. El adulto sabe que los ordenadores no interpretan, únicamente decodifican; no extrapolan ni infieren injustificadamente como acostumbran a hacer los humanos. Una de las soluciones, la que persigue la investigación sobre inteligencia artificial, es diseñar ordenadores más "inteligentes» elaborando una base de conocimientos más rica que guíe la interpretación. Otra solución consiste en preparar a las personas, niños incluidos, para que expliciten más sus significados, menos abiertos a interpretación o malinterpretación. El ordenador permite un feedback inmediato sobre el modo en que ha interpretado una instrucción por tanto, establece un nuevo estándar de precisión que cualquier persona que trabaje con él descubre pronto. Este estándar no es imposible de alcanzar. Recuérdese que la alfabetización nos ha hecho recorrer la mitad del camino hacia esa meta.

¿Es posible enseñar a los niños a reconocer la diferencia entre lo que realmente se ha dicho y la interpretación que ellos tienden a hacer, con mayor facilidad cuando se trate de un ordenador que cuando se trate de una persona? Como ya he dicho, los niños asumen con facilidad que las personas, ven, oyen, infieren e interpretan lo mismo que ellos; tienen dificultades para comprender que los demás podrían hacer una interpretación diferente. Pero, ¿asumirían esto mismo en el caso de un ordenador? Parece razonable suponer que los ordenadores no invitan a la presuposición de equivalencia con otras mentes que hacen los interlocutores humanos. Pero esa es una hipótesis que tendría que investigarse. Yo presiento que los niños no asumirán, al menos así como así, que el ordenador haga las mismas interpretaciones e inferencias que ellos hacen y que normalmente atribuyen a otras personas. Tendrán que remitirse a lo que se dijo exactamente, a lo que está explícitamente representado en el lenguaje. En concreto, en caso de fracaso comunicativo, los niños tal vez culpen al mensaje más fácilmente cuando trabajen con un ordenador que cuando trabajen con otro ser humano. Reconocerán que los ordenadores sólo descifran lo que está ahí, no lo "leído entre líneas». Además, el que los niños reconozcan que hay ciertos tipos de mensajes que pueden ser ambiguos para el ordenador, puede hacerles considerar la ambigüedad de su propio lenguaje oral y escrito. Por consiguiente, aprender a utilizar un ordenador podría contribuir a la competencia del niño en esta importante distinción conceptual.

Si de una investigación de este tipo se obtienen los resultados predi- 
chos, sabremos algo más tanto sobre ordenadores como sobre la comprensión del lenguaje en los niños. Al enfrentarse con los ordenadores, como sucede en el caso de la lengua escrita, los niños deben distinguir la intención de lo que realmente expresa el mensaje o el texto, y deben tratar de expresar su intención plenamente en el mensaje o en el texto. Es decir, el ordenador depende de la precisión del lenguaje en mayor grado aún que la lengua escrita. Sabremos que es posible enseñar a los niños a hacer esta distinción más fácilmente con un ordenador. Podemos deducir que obtiene más éxito porque no son tan propensos a atribuir a los ordenadores los mismos pensamientos que se atribuyen a sí mismos y a otras personas. En efecto, puede darse el caso de que la retroalimentación inmediata y directa, y la incapacidad del ordenador para «leer» sus intenciones desanimen al niño a la hora de atribuirle cualquier modalidad de pensamiento.

Nótese que esta sugerencia se opone frontalmente a lo que Turkel (1984) expuso en su popular libro sobre ordenadores y niños. Turkel sugiere que el trabajar con el ordenador lleva al niño a atribuir estados mentales tanto al ordenador como a sí mismo. Yo creo que los niños comprenderán rápidamente que el ordenador no puede interpretar, leer entre líneas o albergar cualquier tipo de estado mental subjetivo. Lejos de fomentar la concepción de subjetividad el ordenador ayuda a erradicar en general este tipo de concepciones.

\section{Modificaciones en las operaciones cognitivas}

En cuanto a las modificaciones de las operaciones mentales que los ordenadores pueden traer consigo, es importante advertir que éstos no son "herramientas del intelecto" en el sentido que lo fue y aún lo es la escritura. Los ordenadores son inteligentes por sí mismos. De hecho, su posible impacto sobre la cognición reside en emparejar sus recursos con los recursos de la mente.

¿Qué aspectos de la competencia intelectual pueden asignarse al ordenador? La reconsideración del impacto de la lengua escrita en la cognición puede ofrecernos algunas indicaciones.

Con la lengua escrita, la función de la memoria ha pasado de centrarse únicamente en la preservación del contenido a centrarse en la organización y la recuperación. Téngase en cuenta cómo el desarrollo de bibliotecas e índices para almacenar y localizar la información cambió esta función cognitiva esencial. Claro que la memoria está también implicada en una sociedad oral. Pero en una sociedad alfabetizada, se hace hincapié en el conocimiento de la estructura lógica de la información, en la medida en que es crucial para localizarla (las categorías relacionadas jerárquicamente llegan a ser más importantes que la memoria narrativa y episódica de los sucesos). Además, los recursos de archivo de una biblioteca son mayores que los del hombre sabio tradicional; lo que se sabe es mucho más de lo que cualquier individuo sabe.

Del mismo modo que la lengua escrita reorganiza y expande la memoria, el ordenador expande la planificación de la acción mucho más allá de lo que cualquier mente sería capaz. Nuestra sociedad ha logrado disponer en su seno de una enorme cantidad de información y de gran parte de la organización de acción planificada, parte de ella en forma de programas de ordenador. Por ello, nuestra cultura y nuestra tecnología posibilitan que el 
hombre llegue a la luna y que se establezcan vastos programas sociales económicos.

Prácticamente todas las modalidades de competencia humana son un producto conjunto de los recursos de la mente y de las potencialidades de estas tecnologías. Casi cualquier forma de cognición humana exige trabajar productiva e imaginativamente con ellas. Intentar caracterizar la inteligencia con independencia de estas tecnologías es un grave error. Al emplear una tecnología en la consecución de un efecto concreto, uno debe hacer distinciones conceptuales - cambiar la base de conocimientos y llevar a cabo ciertas operaciones específicas-, cambiar las funciones cognitivas para explotar al máximo los recursos tecnológicos. Volviendo a nuestro ejemplo anterior, una persona puede aprender a programar, escribir y a hablar con más claridad si el ordenador lo exige. Por el contrario, si las demandas son excesivas, se puede modificar la tecnología de manera que responda a los hábitos del usuario. Pero ni las propiedades del ordenador ni las propiedades de la mente son entidades estáticas. La invención tecnológica es una de las más importantes para descubrir las propiedades de la mente, que aún están por determinar.

\section{Conclusión}

Por lo que respecta a la cognición infantil y al uso creciente del ordenador en la escuela y en la sociedad, incluso el ordenador más sencillo exige un grado de claridad en el lenguaje mayor que el requerido por el lenguaje de la conversación cotidiana. Los ordenadores nunca comprenden enunciados ambiguos y nunca leen entre líneas, mientras que los humanos son capaces de hacerlo y de hecho es precisamente lo que hacen. $\mathrm{Al}$ aprender a manejar ordenadores, los niños aprenderán a hacer sus significados plenamente explícitos. El lenguaje escrito persigue este mismo objetivo a menor escala. Por tanto, el ordenador requerirá tanto una concepción de la ambigüedad de mensajes y textos, como un criterio definido para determinar si se ha conseguido el objetivo de la precisión del significado.

Visto desde esta perspectiva, el ordenador simplememente aumenta, en orden de magnitud, el requisito de hacer explícitos los significados, un requisito que surgió al dar una semántica a los sistemas de comunicación y que se ha mantenido al hacer dicha semántica más explícita y elaborada por medio de la escritura. Lejos de haber quedado obsoletas, las competencias de lectura y escritura son básicas a la hora de trabajar frente a un ordenador. Ser inteligente en esta sociedad de usuarios de ordenadores quiere decir ser hábil en hacer explícitos los propios significados. En el siglo XX, en plena era de la informática, estamos ganando la batalla que el siglo XVI, la era de la escritura, aspiraba a ganar: elaborar significados claros y precisos.

\section{Referencias}

BEAL, C. y Flavell, J. (en preparación). "Young speakers' evaluations of their listeners comprehension in a referential communication task $*$.

Evans-PritchaRD, E. (1937). Witchcraft, oracles and magic among the Azande, Oxford, Clarendon.

GoODY, J. (1977). The domestication of the savage mind. Cambridge: Cambridge University Press. 
HAVELOCK, E. (1982). The literate revolution in Greece and its cultural consequences. Princenton: Princenton University Press.

McLuhaN, M. (1962). The Gutenberg galaxy. Toronto: Toronto University Press.

OLSON, D. (1976). Culture, technology and intellect. En The nature of intelligence, Potomac, MD, Erlbaum.

OLSON, D. (en preparación). The world on paper.

OLSON, D. y TORRANCE, N. (1983). Literacy and cognitive development: A conceptual transformation in the early school years. En S. Meadows (comp.): Issues in chilhood cognitive development, Londres, Methuen.

OONG, W. (1982). Orality and literacy. The technologizing of the world, Nueva York, Methuen.

Robinson, E.; Goelman, H. y Olson, D. (1983). Children understanding of the relation between expressions (what was said) and intentio (what was meant). British Journal of Developmental Psychology, 1, 75-86.

ROBINSON, E. y ROBINSON, W. (1982). Knowing when you dont't know enough: Children's judgements about ambiguous information, Cognition, 12, 267-280.

TURKEL, S. (1984). The second self: Computers and the buman spirit, Nueva York, Simon and Schuster.

\section{Datos sobre el autor}

David $R$. Olson trabaja en el Ontario Institute for Studies in education, 252

Bloor Street West, Toronto, Ontario M4E2L3, Canadá, y colabora en el programa McLuhan de cultura y tecnología de la Universidad de Toronto. Su área de especialización gira en torno a las implicaciones cognitivas de la literidad.

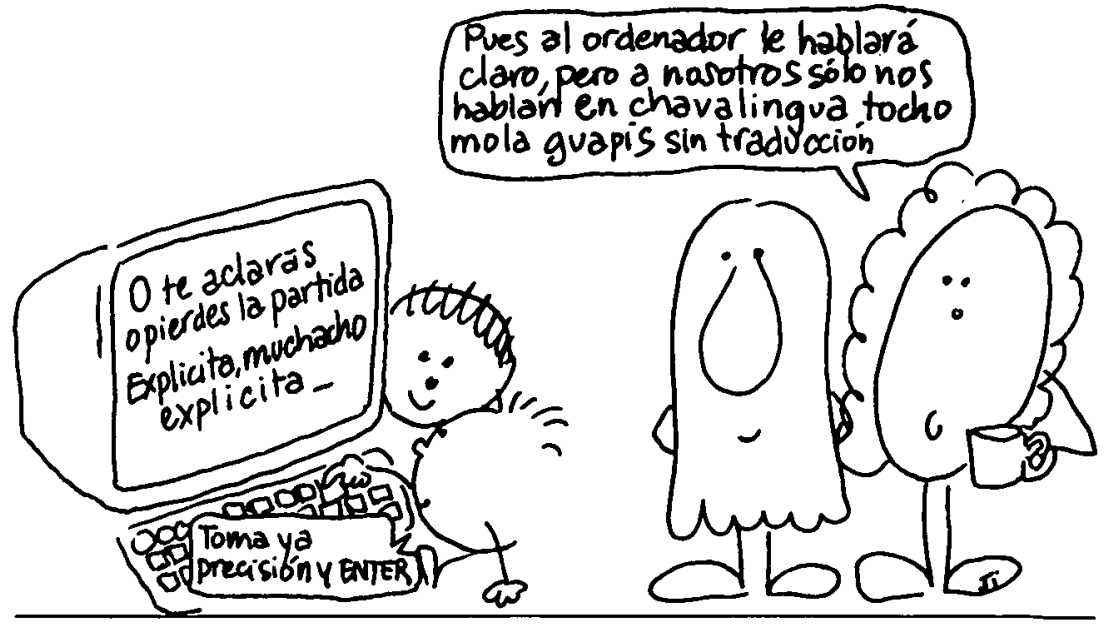

